\title{
ELEMENTS OF WAVE THEORY
}

\author{
R. L. Wlegel and J. W Johnson \\ Respectively, Institute of Engineering Research, and Division \\ of Mechanical Engineering \\ University of Calıfornia \\ Berkeley, Calıfornia
}

\section{INTRODUCTION}

The first known mathematical solution for finite height, periodic waves of stable form was developed by Gerstner (1802). From equations that were developed, Gerstner (1802) arrived at the conclusion that the surface curve was trocholdal in form. Froude (1862) and Rankine (1863) developed the theory but in the opposite manner, i.e., they started with the assumption of a trochoidal form and then developed their equations from this curve. The theory was developed for waves in water of infinite depth with the orbits of the water particles being circular, decreasing in geometrical progression as the distance below the water surface increased in arithmetical progression. Recent experiments (Wiegel, 1950) have shown that the surface profile, represented by the trocholdal equations (as well as the first few tems of Stokes' theory), closely approximates the actual profiles for waves traveling over a horizontal bottom. However the theory necessitates molecular rotation of the particles, while the manner in which waves are formed by conservative forces necessitates irrotational motion.

The first satisfactory treatment of two dimensional wave motion in water of arbitrary depth was given by Ia Place (1776) for waves of small amplitude. Airy (1845) developed an irrotational theory for waves traveling over a horizontal bottom in any depth of water. This theory was developed for waves of very small height. Alry (1845) showed that the velocity of propagation of the wave form was dependent upon the wave length as well as upon the water depth.

Stokes (1847) presented an approximate solution for waves of finite height which satisfied the boundary conditions of waves in water of uniform depth and, in addition, required irrotational motion. The series was to the third approximation for finite depths, or to the fifth approximation for infinite depths, but there was no proof of their convergence. The most interesting features of the solution, apart from the irrotational motion, were, first, the dependency of the wave velocity upon wave helght as well as upon wave length and water depth and, second, the fact that orbital motion of the particles was open rather than closed, indicating a mass transport in the direction of wave travel. Experiments (Mitchim, 1940) have shown both of these findings to be correct.

Levi-Civita (1925) proved that Stokes' series was convergent for "deep-water" waves and Struik (1926) proved that it was convergent for "shallow-water" waves.

Reynolds (1877) and Rayleigh (1877) worked on the problem of the difference between the energy transmission velocity of a wave group and the velocity of the wave form. They concluded that the energy of the group of waves was propagated with a velocity less than that of the individual waves. In deep water, the "group" velocity was found to be one-half the wave velocity.

The problem of the maximum steepness (the ratio of the wave height to its length) that a wave could attain without breaking was worked on by Stokes (1847), Michel1 (1893), and Havelock (1918). Their conclusions were in close agreement. A crest angle of 120 degrees, or a steepness of $H / L=0.142$, * was found to be the theoretical limit.

Recently, many field and laboratory studies, as well as analytical studies, have been made. These observations, together with the mathematical studles, lead

* See list of symbols. 


\section{COASTAL ENGINEERING}

to the conclusion that Stokes' irrotational theory represents the natural phenomena more closely than the other theories.

Waves in nature vary considerably in height and period over a relatively short length of time at any point of observation. In a generating area, the wave characteristics show the maximum variability; however, even after the waves have passed into a region of relative calm, considerable variations in wave characteristics exist. In theoretical problems, such variability cannot be treated mathematicaliy and certain idealized conditions must be assumed. Accordingly, the first step in the analysis of oscillatory waves is to study the behavior of single wave trains of uniform period and amplitude as they progress in water of constant depth. Present-day wave theory deals with periodic waves of stable form in which all elements of the wave profile advance with the same velocity relative to the undisturbed water. Complete development of the various analyses of Lamb (1932), Stokes (1847), Gerstner (1802), and others are not presented herein as they are readily available in the original references.

\section{WAVES OF SMALI AMPLITUDE}

If waves are of small amplitude compared to their length and to the depth of the water, the wave profile closely approximates a sine curve. The equation for motion (Lamb, 1932), considering both gravity and surface tension, is:

$$
\mathrm{c}^{2}=\left(\mathrm{gL} / 2 \pi+2^{\pi \sigma / \rho L}\right) \tanh 2^{\pi \mathrm{d} / L}
$$

For water deeper than one-half the wave length, $\tanh 2 \pi \mathrm{d} / \mathrm{L}$ is almost equal to 1 and the equation reduces to:

$$
\mathrm{C}_{0}^{2}=\mathrm{gI_{0 }} / 2 \pi+2 \pi \sigma / \rho \mathrm{I}_{0}
$$

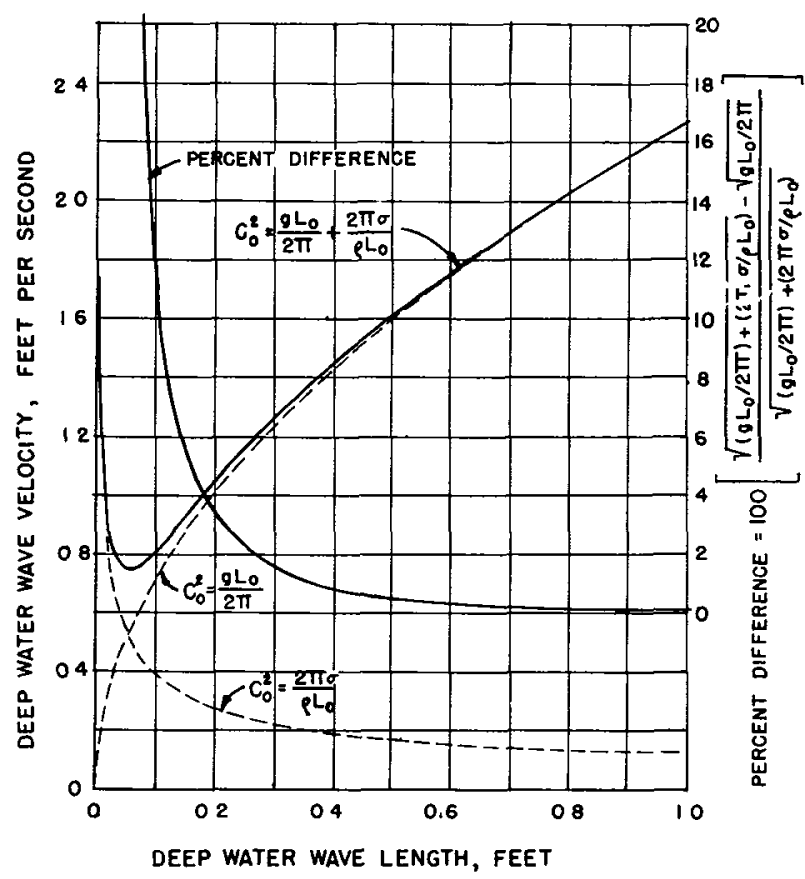

Fig. 1. Effect of surface tension on deep water wave velocity in fresh water at $70^{\circ} \mathrm{F}$.
The relative effects on velocity of the gravity and the surface tension components for deepwater waves are presented in Fig. 1 . Experimental data by Chinn (1949) and Kaplan (1950) verifies the equation. It can be seen that for any wave over a foot in length, the effect of surface tension may be neglected. In practice, these small waves are usually called ripples as distinguished from the longer waves.

Neglecting the effect of surface tension, the equation for velocity of propagation of gravity waves (Airy, 1845; Lamb, 1932)

$$
\mathrm{c}^{2}=(\mathrm{gL} / 2 \pi) \tanh 2 \pi \mathrm{d} / \mathrm{L}
$$

and for "deep-water:"

$$
c_{0}{ }^{2}=g L_{0} / 2 \pi
$$

or, in English units:

$$
\mathrm{C}_{0}{ }^{2}=5.12 \mathrm{~L}_{0}
$$

Since the relationship between length, period and velocity of all periodic wave phenomena is defined by:

$$
\mathrm{I}=\mathrm{CT}
$$




\section{ELEMENTS OF WAVE THEORY}

It follows that:

$$
\mathrm{L}_{0}=g \mathrm{~T}^{2} / 2 \pi
$$

or, in English units for "deep-water.":

$$
L_{0}=5.12 \mathrm{~T}^{2}
$$

Actually, there is no abrupt change from "deep" to "shallow" water. The effect of depth of water on the wave characteristics is gradual, and waves in any finite depth of water are affected by the depth. The depths for which the simplified equations are no longer applicable depends upon the degree of accuracy desired in calculations. The custom has developed, for most engineering studies, to call water which is deeper than one-half the wavie length "deep-water" and water which is less than half the wave length "shallow water."

At the other extreme, "very shallow water," tanh $2 \pi \mathrm{d} / \mathrm{L}$ approaches the value of $2 \pi d / L$ and equation 3 becomes:

$$
\mathrm{c}^{2}=\mathrm{gd}
$$

the well-known equation for very long low waves This equation also holds for waves of finite height. Fig. 2 shows the relationships between wave period, wave velocity, and depth of water. Fig 3 shows the relationships of wave period, wave length and depth of water

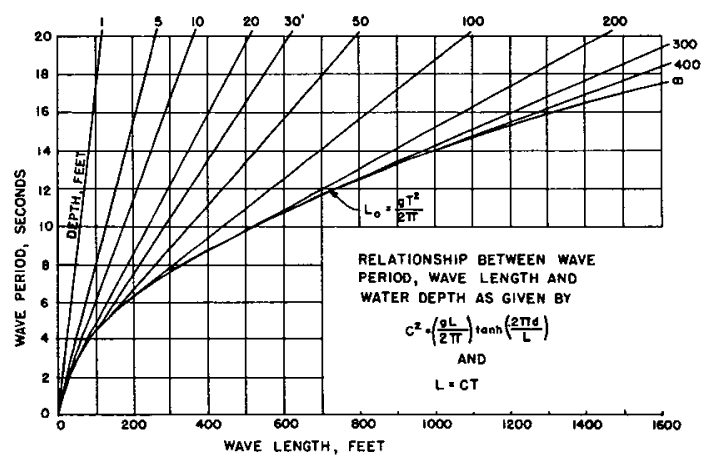

Fig. 2

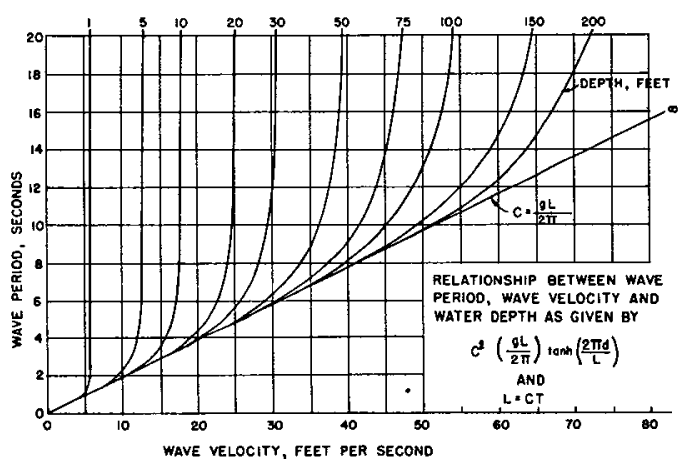

Fig. 3

Surface Profile. The surface curve for waves of small amplitude as given by this theory is the sinusoidal equation:

$$
y=(H / 2) \cos 2 \pi(t / L-x / L)
$$

Orbital Motion. The motion of the individual particle is elliptical (Fig. 4a).

a. SHALLOW WATER WAVE

$$
\begin{aligned}
& L=10 \mathrm{FT} \\
& H=02 \mathrm{FT} \\
& d=10 \mathrm{FT}
\end{aligned}
$$

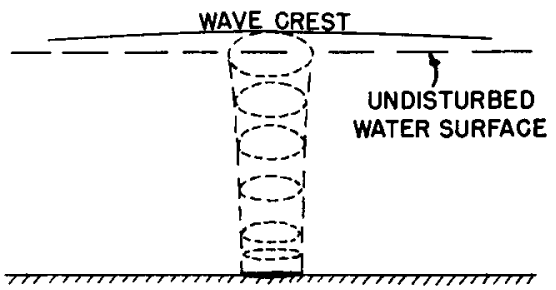

вотTOM b DEEP WATER WAVE

$L=10 \mathrm{FT}, \quad H=2 \mathrm{FT}, \quad d=10 \mathrm{FT}$

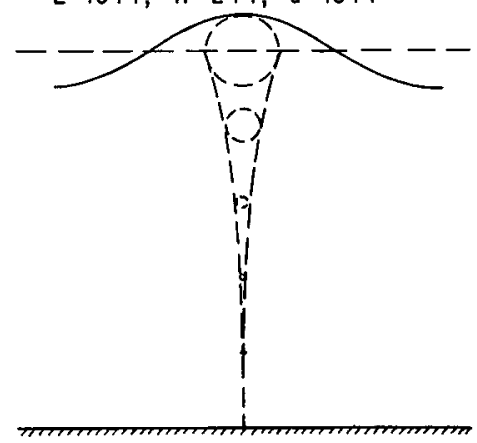

Fig. 4 


\section{COASTAL ENGINEERING}

The horizontal and vertical displacements from its mean position, a distance $z$ (measured negatively downward) below the still-water surface, are:

$$
\begin{aligned}
& \xi=\frac{1}{2} H \frac{\cosh 2 \pi(d+z) / L}{\sinh 2 \pi d / L} \cos 2 \pi(x / L-t / T) \\
& \eta=\frac{1}{2} H \frac{\sinh 2 \pi(d+z) / L}{\sinh 2 \pi d / L} \sin 2 \pi(x / L-t / T)
\end{aligned}
$$

From these equations it can be seen that the semi-orbital amplitudes of the subsurface particle's motions are:

$$
\begin{aligned}
& a^{\prime}=\frac{1}{2} H \frac{\cosh 2 \pi(d+z) / L}{\sinh 2 \pi d / L} \\
& b^{\prime}=\frac{1}{2} H \frac{\sinh 2 \pi(d+z) / L}{\sinh 2 \pi d / L}
\end{aligned}
$$

with the ratio of the orbital amplitudes (Fig. 5) being:

$$
\frac{b^{\prime}}{a^{\prime}}=\tanh 2 \pi(d+z) / L
$$

Recent experiments (Morison, 1948) have verified these equations (Figs. 6 and 7) except that, in addition, there is some mass transport. The full amplitude of the orbital motion at the surface $\left(2 a_{s}^{\prime}\right.$ and $\left.2 b_{s}^{\prime}\right)$ may be expressed as:

$$
\begin{aligned}
& 2 \mathrm{a}_{\mathbf{s}}{ }^{\prime}=\mathrm{H} \operatorname{coth} 2 \pi \mathrm{d} / \mathrm{L} \\
& 2 \mathrm{~b}_{\mathbf{S}}{ }^{\prime}=\mathrm{H}
\end{aligned}
$$

When the equations are converted into their exponential form, it is found that as the water depth approaches infinity:

$$
\begin{aligned}
& a^{\prime} \longrightarrow \frac{1}{2} \mathrm{He}^{2 \pi \mathrm{z} / \mathrm{L}} \\
& \mathrm{b}^{\prime} \longrightarrow \frac{1}{2} \mathrm{He}^{2 \pi \mathrm{z} / \mathrm{L}}
\end{aligned}
$$

However, the horizontal and vertical semi-amplitudes approach these limiting values at different rates with respect to $z$ (Fig 5). So, although the orbital motion near the surface becomes nearly circular in shape very rapidly as the depth of water increases, the orbital paths become flatter and flatter with increasing distance below the surface until, at the bottom, the vertical motion is zero and so the particle moves back and forth with a purely horizontal motion. Only when the water depth becomes "Infinite" are all the particle paths circular. Fig. 8a shows the vertical amplitude of oscillation for various depths and wave lengths, and Fig. $8 b$ shows the horizontal amplitude of oscillation for various depths and wave lengths.

By differentiating the horizontal and vertical orbital displacements with respect to time, the horizontal and vertical components of the water particle velocities occupying an average position at a distance $z$ below the center of the surface particle (this neglect of second order quantities appears to be allowable) are found to be:

$$
\begin{aligned}
& u_{z}=\frac{\partial Y}{\partial t}=\frac{\pi \cdot H \cosh 2 \pi(d+z) / L}{T \sinh 2 \pi d / L} \sin 2 \pi(x / L-t / T) \\
& v_{z}=\frac{\partial \eta}{\partial t}=\frac{-\pi H \sinh 2 \pi(d+z) / L}{T \sinh 2 \pi d / L} \cos 2 \pi(x / L-t / T)
\end{aligned}
$$

with the average velocities over one-half their cycle being:

$$
\begin{aligned}
& \left(u_{z}\right) \text { ave. }= \pm \frac{2 H \cosh 2 \pi(d+z) / L}{T \sinh 2 \pi d / L} \\
& (v z)_{a v e .}= \pm \frac{2 H \sinh 2 \pi(d+z) / L}{T \sinh 2 \pi d / L}
\end{aligned}
$$




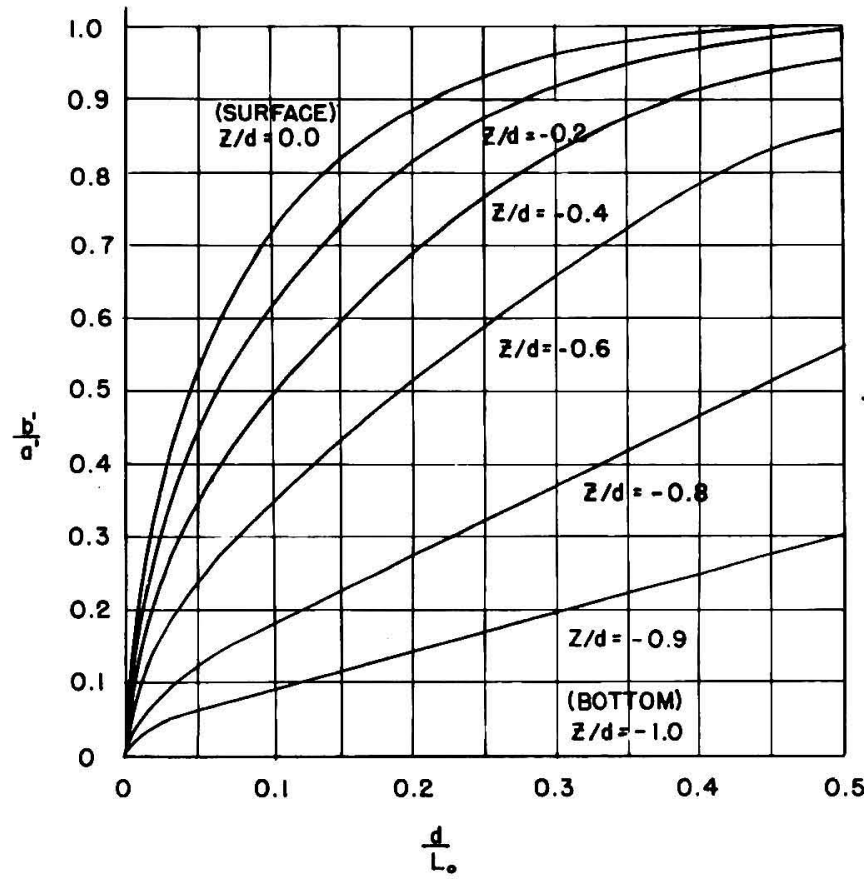

Fig. 5

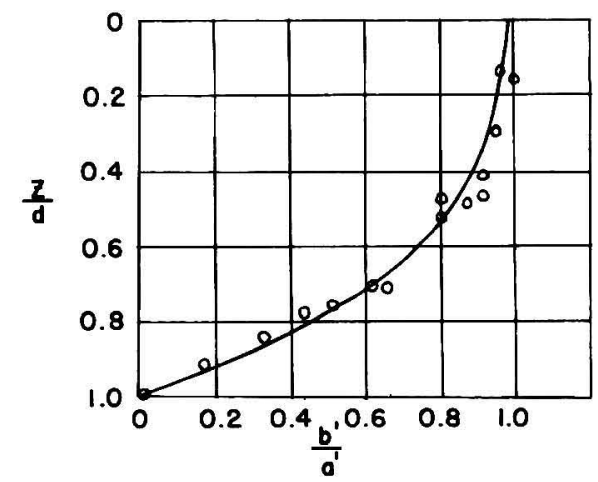

a. Comparison of the ratios of measured orbit axes with theory for $d / L_{0}=0.39$

F1g. 7

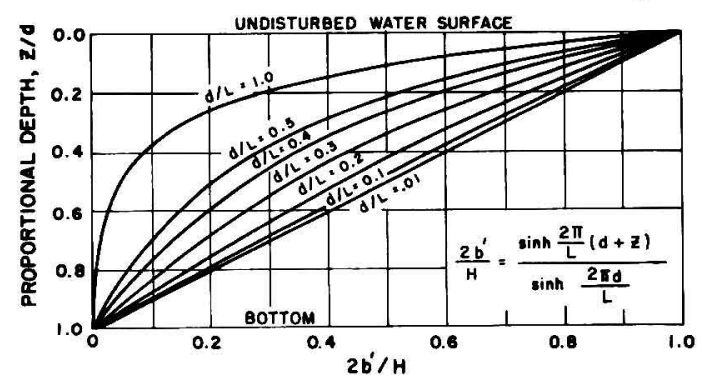

RATIO OF VERTICAL AMPLITUDE OF ORBIT TO WAVE HEIGHT

a. Vertical amplitude of oscillation for proportional depth related to fraction of wave height.

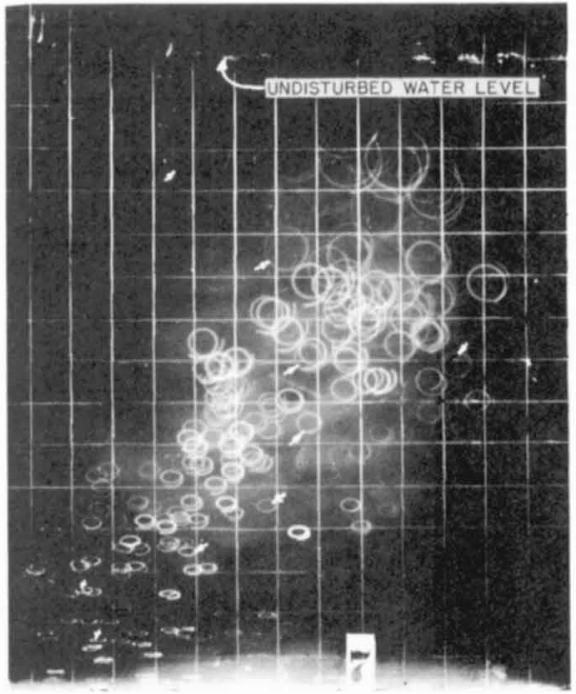

Fig. 6. Photograph of water particle orbits for a wave with the following dimensions.

$d=2.50$ feet, $H=0.339$ feet, $L=6.42$ feet, $T=112$ seconds and $d / L_{0}=0.39$ 


\section{COASTAL ENGINEERING}

and their maximum orbital velocities being,

$$
\begin{aligned}
& \left(u_{z}\right)_{\max .}=\frac{\pi H \cosh 2 \pi(d+z) / L}{T \sinh 2 \pi d / L} \\
& \left(v_{z}\right)_{\max .}=\frac{\pi H \sinh 2 \pi(d+z) / L}{T \sinh 2 \pi d / L}
\end{aligned}
$$

An example of the manner in which the maximum horizontal component of orbital velocity varies with the period is shown in Fig. 9. These values are for a particle on the ocean bottom.

Energy of Waves. The kinetic energy per unit width (along the crest) for a wave is the summation of the kinetic energy of the particles in motion. For a wave of sinusoldal form in deep water, this is given by,

$$
E_{k}=w_{o} H_{o}^{2} / 16
$$

The potential energy per unit width for a wave is computed from the elevation or depression of the water from the undisturbed level and is given by,

$$
E_{p}=w_{o} H_{o} 2 / 16
$$

It can be seen that half of the energy of a wave is kinetic and half potential. The total energy is expressed by,

$$
E=\mathrm{wL}_{\mathrm{O}} \mathrm{H}_{\mathrm{O}} 2 / 8
$$

which, when combined with Equation $6 a$, gives,

$$
E=w g T^{2} H_{0} 2 / 16 \pi
$$

Effect of Viscosity. The effect of viscous damping of water waves of small amplitude of sinusoidal form has been studied mathematically by Lamb (1932) for waves in deep-water and by Hough (1896) for waves in any depth of water (assuming the bottom to be perfectly smooth). The modulus of decay, $t_{\nu}$, (the time necessary for the wave height to be reduced in the ratio of $e: 1$ ) is given by,

$$
t_{\nu}=L^{2} / 8 \pi^{2} \nu
$$

It can be seen from Fig. I0 that extremely short (capillary) waves die out rapidly but that the damping is very small for waves of any appreciable length.

Sub-Surface Pressures. With the development of the pressure type wave recorder (Folsom, 1949; Isaacs and Wlegel, 1950), it became necessary to utilize the equations for pressure at any point beneath the water surface. The solution (Lamb, 1932) for an incompressible, nonviscous fluid is,

$$
K=[\cosh 2 \pi d L(1-z / d)] / \cosh 2 \pi d / L
$$

where $K$, the sub-surface pressure response factor, is the ratio of the pressure at any depth below the water surface and the pressure at the surface. The ratio of the distance below the surface to the water depth is known as the proportional

- depth. This can be represented in dimensionless form as shown in Fig. 11. Tabulated values have been published by the Beach Erosion Board (Wiegel, 1948). Experiments (Folsom, 1947) show that this approximates the case for waves of finite height. However, for waves of finite height, the measured pressures were about ten percent lower than the theory (for very small waves) predicts.

\section{WAVES OF FIINITE AMPLITUDE}

Experiments (Beach Erosion Board, 1941; Mortson, 1951; Wiegel, 1950) have shown that the equations for waves of small amplitude continue to be valid, as far 


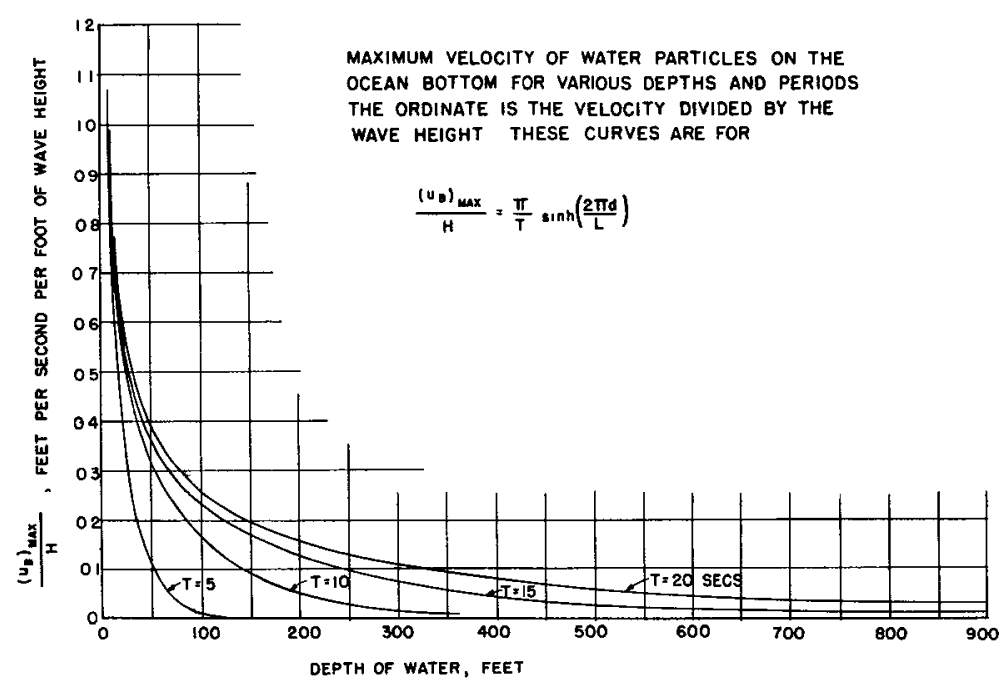

Fig. 9

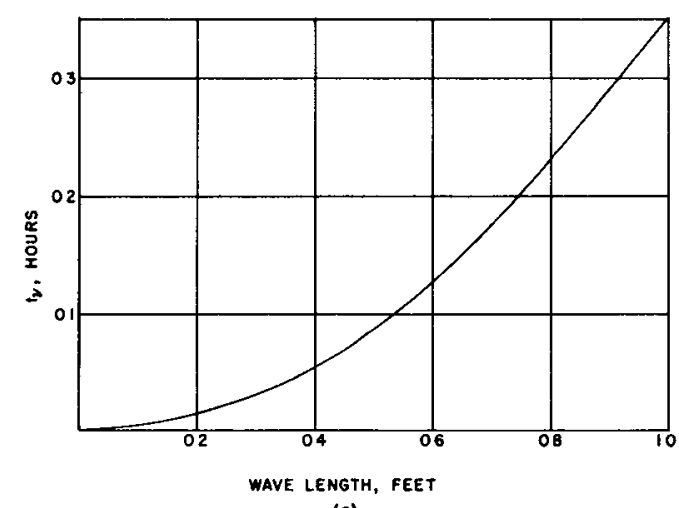

(q)

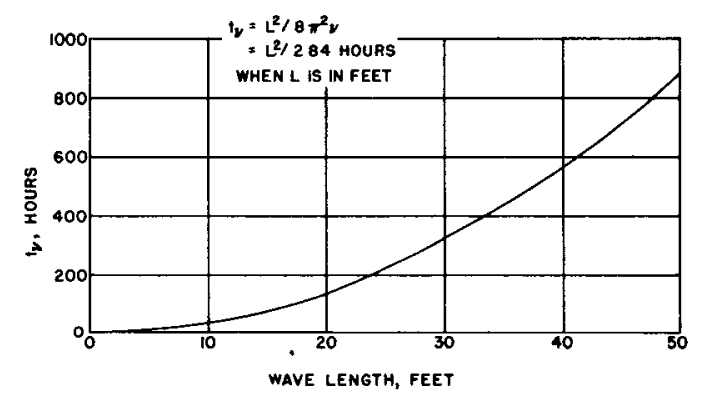

(b)

Relationship between modulus of decay due to viscous damping and wave length. Fig. 10

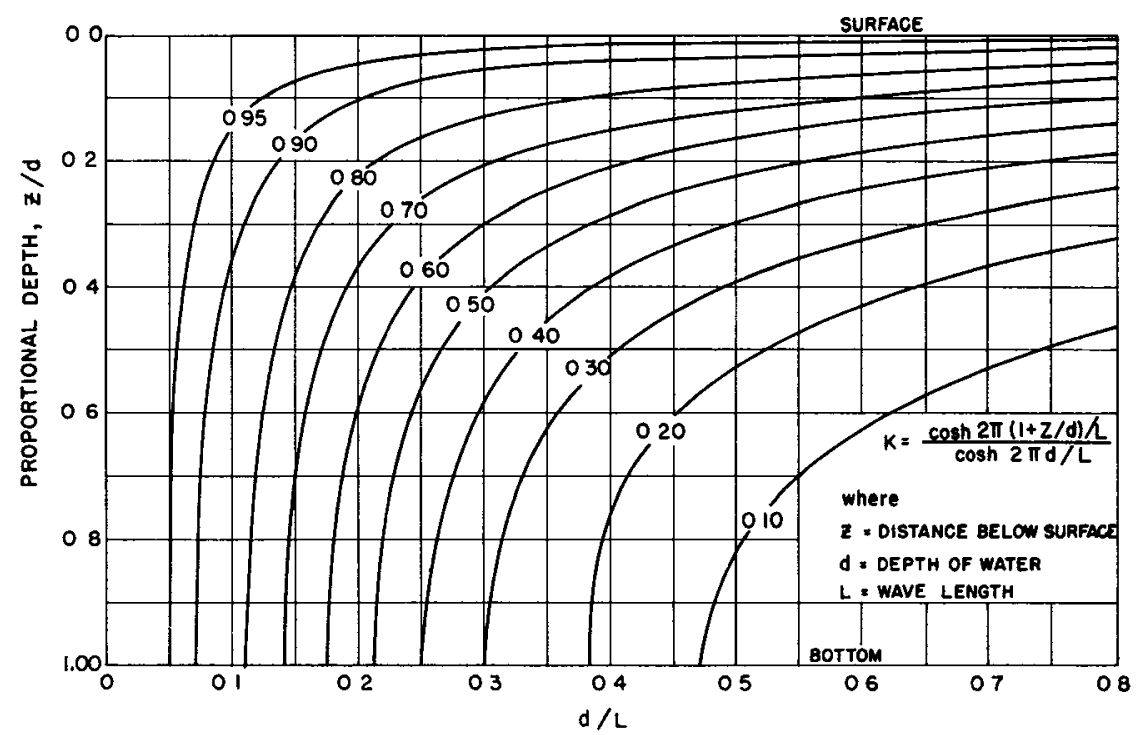

Fig. 11. Pressure response factor. 


\section{COASTAL ENGINEERING}

as engineering applications are concerned, for waves of appreciable height. It has also been observed that the very long, low ocean swell from distant storms are approximately sinusoldal in deep-water. However, for waves of greater height, theory indicates that certain corrections are necessary.

Two theorles have been developed for waves of finite height. The first theory, developed by Gerstner (1802) and later by Froude (1862) and Rankine (1863), is known as the trochoidal theory. This theory has been used widely by naval architects and engineers in their studies. The second theory, developed principally by Stokes (1847) and later by Struik (1926) and Lev1-Civita (1925), is more difficult to apply but it predicts certain results that have been experimentally verified which are not predicted by the trochoidal theory.

Trocholdal Theory - Infinite Water Depth. The trocholdal theory (Gerstner, 1802), the first theory to be developed for waves of finite height, is often used for engineering calculations. One reason for its use is the ease with which the equations may be used. It appears to represent the actual wave profiles as well as actually satisfying the pressure conditions at the surface and the continuity conditions. However, it requires rotation of the particles and does not predict any mass transport in the direction of wave propagation, while observations (Mitchim, 1940; Beach Erosion Board, 1941) show that there is mass transport. This theory, developed for waves in water of infinite depth, has been well presented by Gaillard (1935).

The equations of the surface profile (Fig. 12a) are,

$$
\begin{aligned}
& x=R \theta-r \sin \theta \\
& y=R-r \cos \theta
\end{aligned}
$$

It can be seen that the wave length, $I_{0}$, is equal to $2 \pi R$, while the wave height, $H_{O}$, is equal to $2 r_{S}$, where $r_{s}$ is the value of $r$ for the surface orbit. In order to plot the equation of wave shape in dimensionless form with the origin of the coordinates at the crest and the vertical dimension measured negatively, downward, these equations may be transformed to:

$$
\begin{aligned}
& \mathrm{x}^{\prime} / \mathrm{L}_{0}=1-\left[(\mathrm{rad} \theta / 2 \pi)-\left(\mathrm{H}_{0} / 2 L_{0}\right) \sin \theta\right] \\
& \mathrm{y}^{\prime} / \mathrm{H}_{0}=1 / 2(1-\cos \theta)
\end{aligned}
$$

Where, $x^{\prime}$ and $y^{\prime}$ are measured from the wave crest. These have been plotted in F1g. 13 with $\mathrm{H}_{\mathrm{O}} / \mathrm{L}_{\mathrm{O}}$ as the parameter. It can be seen that as $\mathrm{H}_{\mathrm{O}} / \mathrm{L}_{\mathrm{O}}$ approaches zero, the curve approaches a sine wave and the surface is nearly that as developed in the Irrotational theory for waves of very small amplitude. are,

The positions of the crest and trough relative to the undisturbed water level

$$
\begin{aligned}
& \text { Height of crest }=\mathrm{H}_{\mathrm{O}}-\left[\mathrm{r}_{\mathrm{s}}-\left(\mathrm{r}_{\mathrm{s}}^{2} / 2 \mathrm{R}\right)\right]=1 / 2 \mathrm{H}_{\mathrm{O}}+\pi \mathrm{H}_{\mathrm{O}}^{2} / 4 \mathrm{~L}_{\mathrm{O}} \\
& \text { Depth of trough }=2 \pi \mathrm{R}\left[\mathrm{r}_{\mathrm{s}}-\left(\mathrm{r}_{\mathrm{s}}{ }^{2 / 2 R}\right)\right] / \mathrm{L}_{\mathrm{O}}=1 / 2 \mathrm{H}_{\mathrm{O}}-\pi \mathrm{H}_{\mathrm{o}} 2 / 4 \mathrm{~L}_{\mathrm{O}}
\end{aligned}
$$

Thus, the crest is more than half the wave height above the undisturbed water level, while the trough is less than half the wave helght below this level. Experiments performed by the Beach Erosion Board (1941) verify these relationships (Fig. 14). It should be noted that they verify the results of the theory of Stokes (1847) as well.

The paths described by the water particles during one cycle are circles with the radil decreasing exponentially with depth (Fig. 12b). This is expressed as,

$$
a^{\prime}=b^{\prime}=r_{s} e^{2 \pi z / L_{0}}=1 / 2 H_{0} e^{2 \pi z / L_{0}}
$$




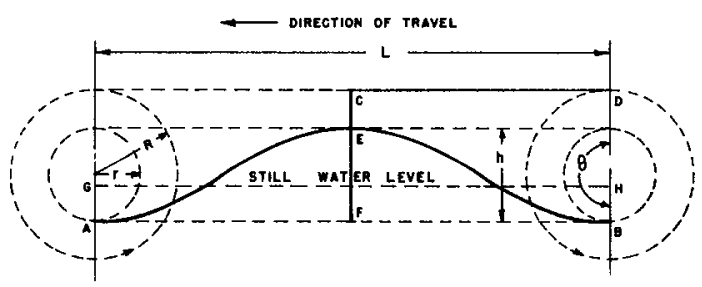

(a)

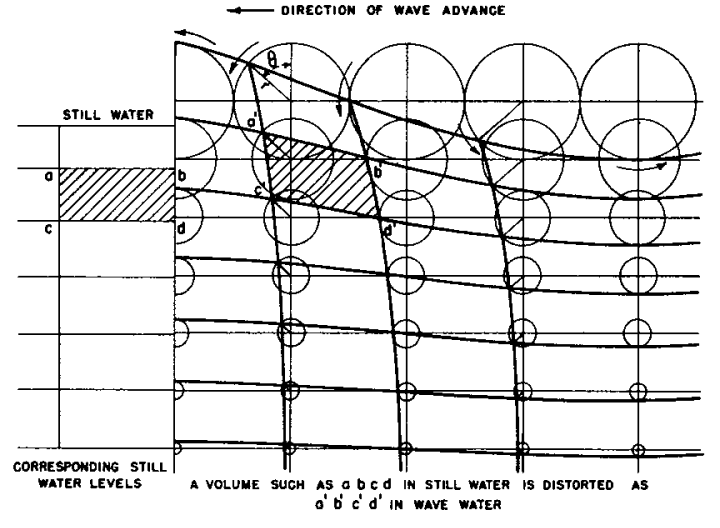

(b)

Fig. 12. Trocholdal Wave.

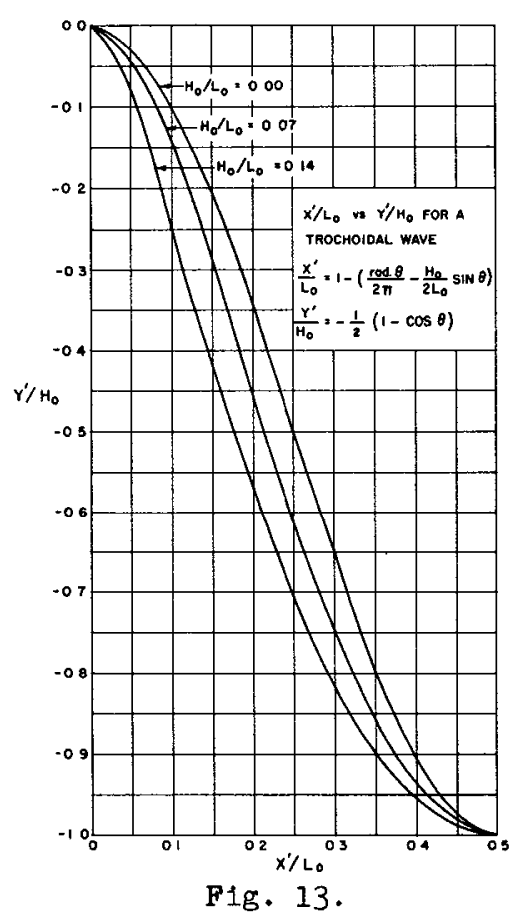

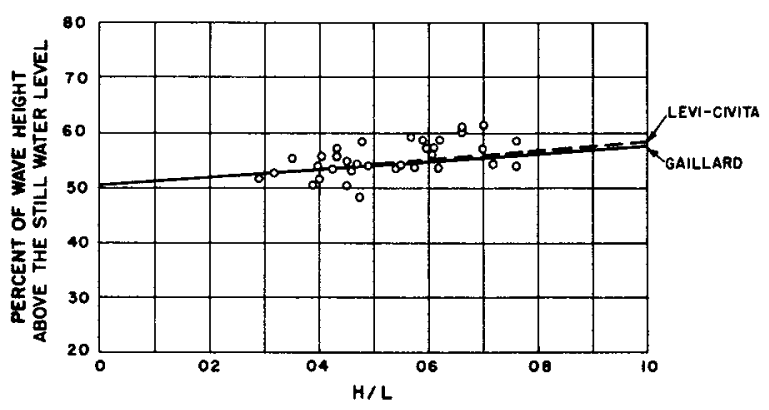

(a) DEEP WATER CONDITIONS - BEACH EROSION BOARD

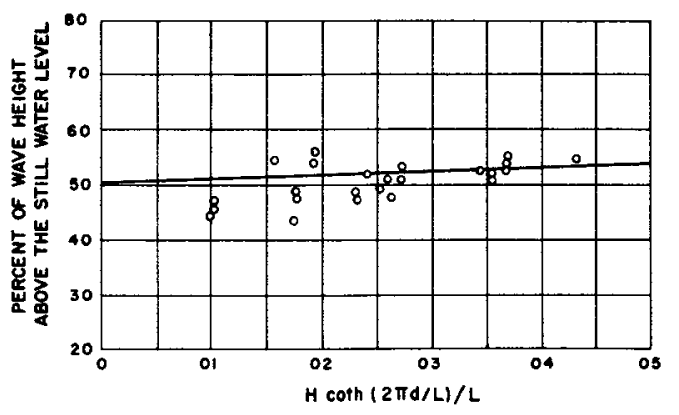

(b) SHALLOW WATER CONDITIONS - UNIVERSITY OF CALIFORNIA

Fig. 14. Percent of wave height above the still water level for water of uniform depth. 


\section{COASTAL ENGINEERING}

The energy of the wave is equally divided between kinetic and potential, with the total energy being,

$$
E=w_{0} \mathrm{H}_{0}^{2}\left[1-1 / 2\left(\pi \mathrm{H}_{\mathrm{O}} / \mathrm{L}_{0}\right)^{2}\right] / 8
$$

Trocholdal Theory - Finite Depth. The trocholdal theory as extended to water of finite depth has been presented by Gaillard (1935) and is widely used. There appears to be no published mathematical work which substantiates the conclusions presented by Gaillard (1935). Perhaps the facts that (a) the wave velocity, orbital velocities and wave shapes as represented in the trochoidal theory were the same as those in the theory of Airy (1845) for waves in deep-water, and (b) other equations of the trochoidal theory reduced to those of Airy (1845) for small amplitudes led Gaillard (1935) to examine the similarities between equations from a reduced (elIiptical) trochoidal theory and the Airy (1845) theory for waves in finite depth. The equations of wave velocity, and orbital velocities and shapes as obtained from the reduced trocholdal theory are the same as those of Airy (1845) for shallowwater waves and for small amplitudes. Other reduced trochoidal equations are almost identical to those of Airy (1845). However, the reduced $t r$ choid theory does not satisfy either the conditions of continuity or dynamical equilibrium except at the trough and crest (Gaillard, 1935) and hence, this theory, although widely used, is not sound.

Gaillard (1935) states that a shallow-water wave differs from a wave in very deep water in that the particle paths are elliptical rather than circular, with the eccentricity of the ellipses depending upon the ratio of the wave length to the depth of water. For a particular length of wave, the eccentricity increases with decreasing water depth so that, in very shallow water, its particle paths are nearly horizontal lines; while the orbits decrease in size with increasing distance below the undisturbed water level with the vertical axes decreasing at a more rapid rate than the horizontal axes until, at the bottom, the vertical motion is zero and the particle moves in a horizontal line. The angular velocity is not constant, but greatest in the vicinity of the trough and crest. It should be noted that this theory predicts that the velocity at the crest of the orbit is the same as the velocity at the bottom of the orbit. Recent experiments performed in the wave channel at the University of California, Berkeley, show that this is not true. The actual crest velocities are greater than the trough velocities.

The following equations, describing the reduced trochoidal surface, were developed and presented by Gaillard (1935) (Fig. 15),

$$
\begin{aligned}
& x=R \theta-a^{\prime} \sin \theta \\
& y=b^{\prime} \cos \theta
\end{aligned}
$$

The velocity of propagation is,

$$
c^{2}=g L b_{s}{ }^{2} / 2 \pi a_{s}^{\prime}=g L(\tanh 2 \pi d / L) / 2 \pi
$$

The equations for the semi-axes of the orbits are,

$$
\begin{aligned}
& b^{\prime}=1 / 2 H[\cosh 2 \pi(d+z) / L] / \sinh 2 \pi d / L \\
& a^{\prime}=1 / 2 H[\sinh 2 \pi(d+z) / L] / \sinh 2 \pi d / L
\end{aligned}
$$

and the ratio of the semi-axes is,

$$
\frac{b^{\prime}}{a^{\prime}}=\tanh 2 \pi(d+z) / L
$$

The total energy of the wave, which is one-half kinetic and one-half potential, is

$$
E=w L H^{2}\left(1-M H^{2} / L^{2}\right) / 8
$$

where $M$, the energy coefficient, is

$$
M=\pi^{2} /\left(2 \tanh ^{2} 2 \pi d / L\right)
$$




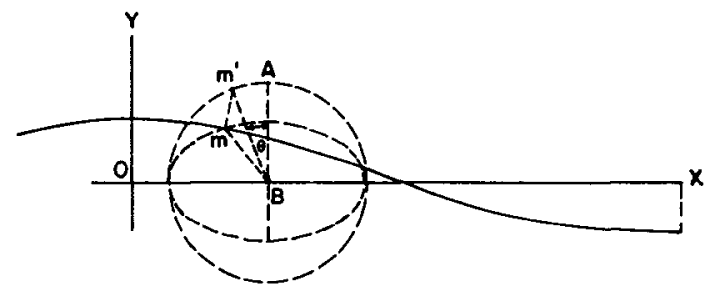

a

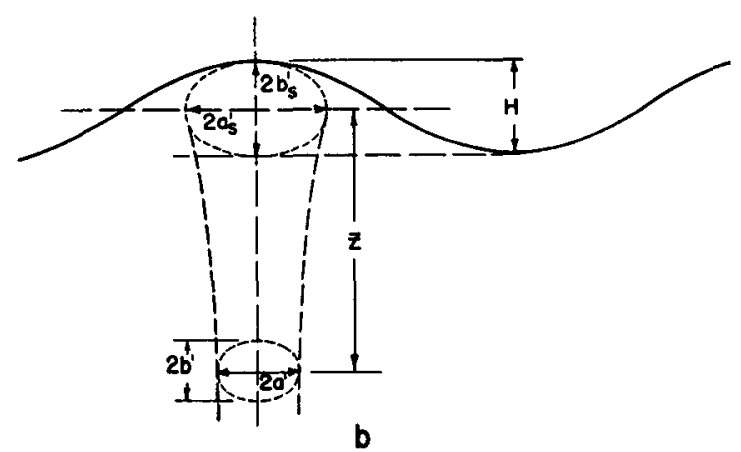

Fig. 15. Shallow water wave, trochoidal theory.

The equations for the shape of the surface profile may be written in a dimensionless form,

$$
x / L=[\operatorname{rad}(\operatorname{arc} \cos 2 y / H) / 2 \pi]-[H \sin (\operatorname{arc} \cos 2 y / H) / 2 \tanh 2 \pi d / L]
$$

This, together with the equation for the displacement of the crest and trough from the undisturbed water level $\left(\mathrm{y}_{\mathrm{s} w 1}\right)$,

$$
\mathrm{y}_{\mathbf{S W} 1} / \mathrm{H}=1 / 2-(\pi \mathrm{H} / 4 \mathrm{~L}) \tanh 2 \pi \mathrm{d} / \mathrm{L}
$$

allows the plotting, in dimensionless form, of the wave profile, or, as $x / L=t / T$, the variation of surface elevation with time. Experiments (Wiegel, 1950) have shown that actual waves are very closely trochoidal in shape (Fig. 16). It should be pointed out that these profiles ( $1 . e$. , for these values of $d / L$ ) are very nearly the same as given to the third approximation by stokes (1847). If the equations for the trochoid are expanded into a series, it can be seen that to the third term it is the same as Stokes' equation as well.

Trochoidal Theory - Rotation. Stokes (1847) has shown that the trocholdal theory necessitates rotation and derives the following expression,

Vorticity $=2 w=\frac{\partial v}{\partial x}-\frac{\partial u}{\partial y}$

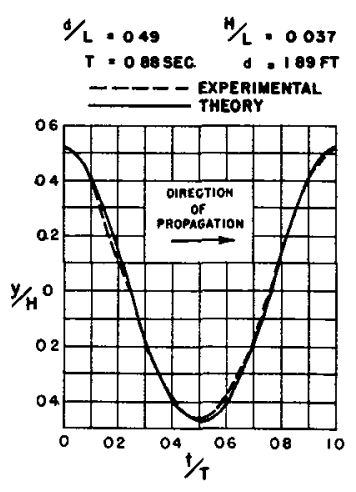

(o)

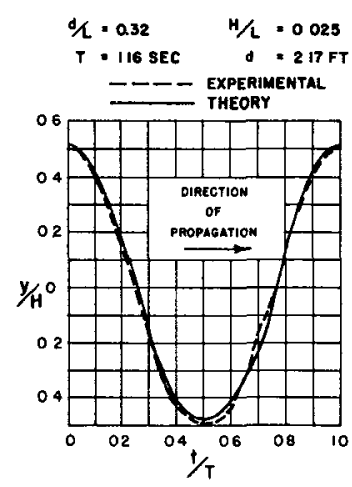

(b)

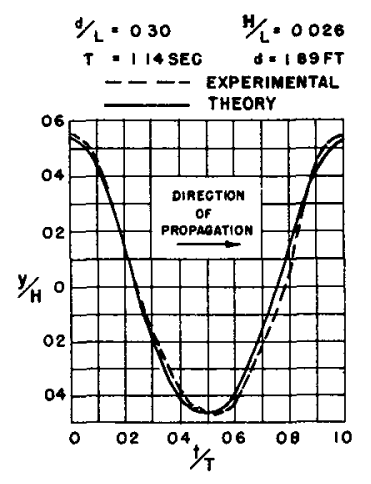

(c)

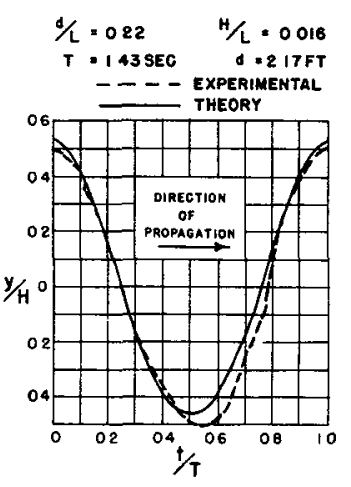

(d)

Fig. 16. Comparison of experimental elevation-time curves with trocholdal theory. 


\section{COASTAL ENGINEERING}

and

$$
\begin{aligned}
& \omega=\frac{-\left(2 \pi^{3} \mathrm{H}^{2} / L^{3}\right) C e^{4 \pi z / L}}{1-(\pi \mathrm{H} / L)^{2} \mathrm{e} 4 \pi \mathrm{z} / L} \\
& U^{\prime}=-\left(\pi \mathrm{H}_{0} / \mathrm{L}_{0}\right)^{2} \mathrm{C}_{\mathrm{O}} \mathrm{e}^{4 \pi \mathrm{z} / \mathrm{L}}
\end{aligned}
$$

where $U^{\prime}$ is the horizontal velocity remaining after wave motion has been destroyed. According to Stokes (1847), "It appears then that in order that it should be possible to excite these waves in deep-water previously free from wave disturbance, by means of pressures applied to the surface, a preparation must be laid in the shape of a horizontal velocity decreasing from the surface downward according to the value $e^{4 \pi z / L}, \ldots "$

Irrotational Theory. The irrotational theory for vaves of finite height in water of uniform depth was developed by Stokes (1847), Rayleigh (1877), Struik (1926), and Levi-Civita (1925). Experimental evidence substantiates the conclusion that this is the theory which most nearly represents actual wave motion.

Stokes (1847) found, to the second approximation, that the velocity of wave propagation is independent of wave height and is the same as the theories of Airy (1845) and Gerstner (1802) (Equation 3). However, to the third approximation,

$$
\mathrm{C}_{\mathrm{H}}^{2}=\frac{\mathrm{gL}}{2 \pi} \tanh 2 \pi d / L\left\{1+\left(\frac{\pi \mathrm{H}}{L}\right)^{2}\left[\frac{\left.2(\cosh 4 \pi d / L)^{2}+2(\cosh 4 \pi d / L)+5\right]}{8(\sinh 2 \pi d / L)^{4}}\right]\right.
$$

which, for deep-water conditions, reduces to:

$$
\left(C_{H}\right)_{O}=\sqrt{\frac{g L_{O}}{2 \pi}\left[1+\left(\frac{\pi H_{O}}{L_{0}}\right)^{2}\right]}
$$

Fig. 17 shows experimental values compared with theoretical values (Morison, 1951). Other experimental work (Beach Erosion Board, 1941) shows approximately the same results. It appears that the experimental error is of the same order of magnitude as the difference between the equations corrected for height and the equations for waves of small amplitude. Because of this, the more simple equation for waves of small amplitude can be used for most engineering calculations.
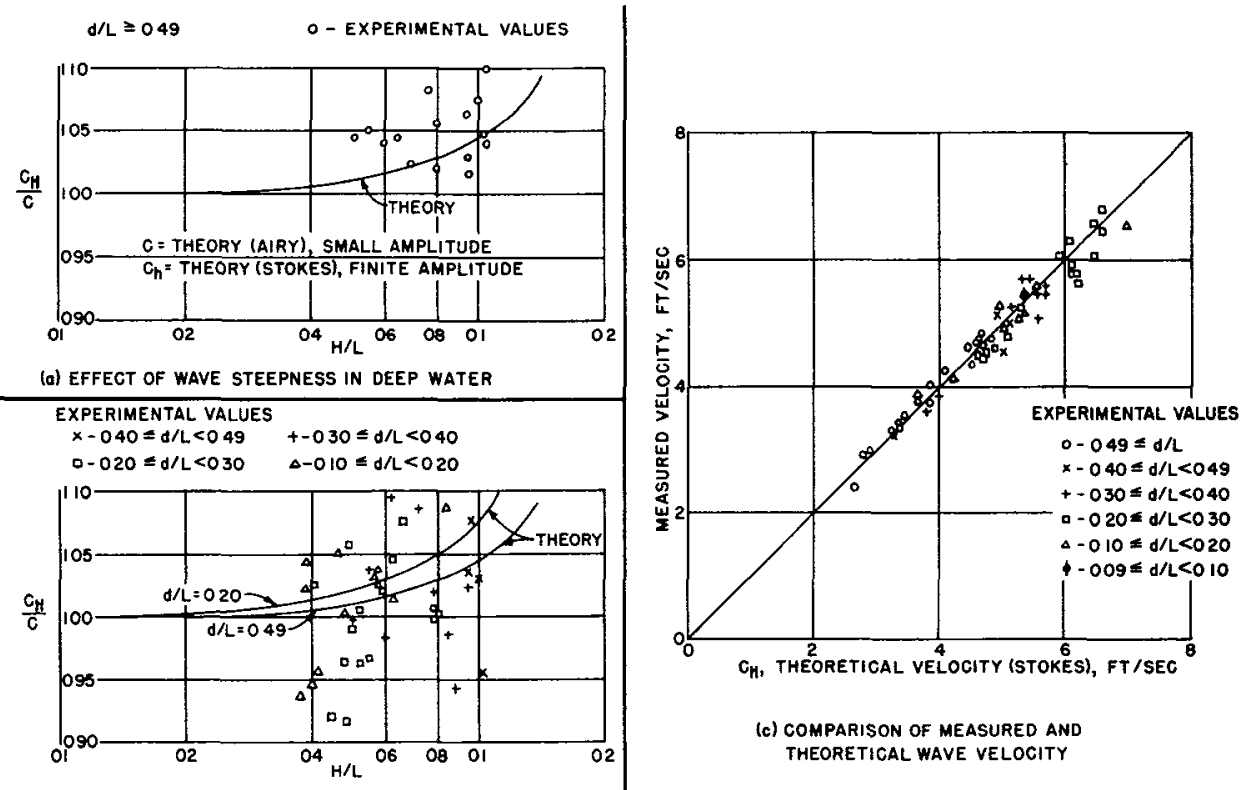
THEORETICAL WAVE VELOCITY

(b) EFFECT OF WAVE STEEPNESS IN SHALLOW WATER

Fig. 17. Velocity of waves of finfte height. 


\section{ELEMENTS OF WAVE THEORY}

The equation for the wave profile, to the third approximation, is:

$y=a \cos 2 \pi x / L+\left(\pi a^{2} / L\right)(\cos 4 \pi x / L)\left[\frac{\left(e^{2 \pi d / L_{+}} e^{-2 \pi d / L}\right)\left(e^{4 \pi d / L}+e^{-4 \pi d / L}+4\right)}{\left(e^{2 \pi d / L}-e^{2 \pi d / L}\right)^{4}}+\right.$

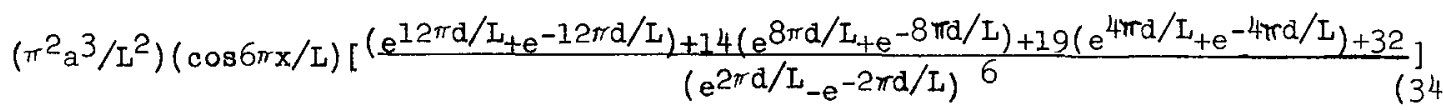

The equations for the horizontal and vertical components of orbital velocities are (according to verbal communication from $R$. A. Fuchs, Institute of Engineering Research, University of California, Berkeley).

$$
\begin{aligned}
\frac{d \xi}{d t}=- & \frac{\pi H}{L} \cdot C\left[\frac{\cosh 2 \pi(d+z+\eta) / L}{\sinh 2 \pi d / L}\right] \cos [2 \pi(x+\xi-c t) / L]+ \\
& 3\left(\frac{\pi H}{L}\right)^{2} C\left[\frac{\cosh 4 \pi(d+z-n) / L}{(\sinh 2 \pi d / L)^{4}}\right] \cos [4 \pi(x+\xi-C t) / L]
\end{aligned}
$$

which, upon expanding, substituting and neglecting terms of third order or higher, becomes,

$$
\begin{aligned}
& \frac{d \xi}{d t}=\frac{-\pi H}{L} \cdot C\left[\frac{\cosh 2 \pi(d+z) / L}{\sinh 2 \pi d / L}\right] \cos [2 \pi(x-C t) / L]+\left(\frac{\pi H}{L}\right)^{2} \cdot \frac{C}{(\sinh 2 \pi d / L)^{2}} . \\
& {\left[-\frac{1}{2}+\frac{3}{4} \frac{\cosh \frac{4 \pi}{L}(d+z)}{(\sinh 2 \pi d / L) 2}\right] \cos [4 \pi(x-C t) / L]+\left(\frac{\pi H}{L}\right)^{2} \frac{C}{2}\left[\frac{\cosh 4 \pi(d+z) / L}{(\sinh 2 \pi d / L)^{2}}\right]}
\end{aligned}
$$

and,

$$
\begin{aligned}
\frac{d \pi}{d t}= & \frac{\pi H}{L} \cdot C\left[\frac{\sinh 2 \pi(d+z+n) / L}{\sinh 2 \pi d / L}\right](\sin 2 \pi(x+\xi-C t) / L)- \\
& \frac{3}{4}\left(\frac{\pi H}{L}\right)^{2} C\left[\frac{\sinh 4 \pi(d+z+\pi)}{(\sinh 2 \pi d / L)^{4}}\right](\sin 4 \pi(x+\xi-C t) / L)
\end{aligned}
$$

which, upon expanding, substituting and neglecting terms of third order or higher, becomes,

$$
\left.\frac{d n}{d t}=\frac{\pi H}{L} \cdot C \frac{\sinh 2 \pi(d+z) / L}{\sinh 2 \pi d / L}(\sin 2 \pi(x-c t) / L)-3 \frac{\pi H}{4}\right)^{2} C\left[\frac{\sinh 4 \pi(d+z) / L}{(\sinh 2 \pi d / L)^{4}}\right](\sin 4 \pi(x-C t) / L)
$$

The equations for particle displacement about their undisturbed positions are,

$$
\xi=\frac{H}{2} \frac{\cosh 2 \pi(d+z) / L}{\sinh 2 m d / I}(\sin 2 \pi(x-C t) / L)-
$$

$\frac{\pi H^{2}}{4 L(\sinh 2 \pi d / L)^{2}}\left[\frac{1}{-2}+\frac{3}{4} \frac{\cosh 4 \pi(d+z) / L}{(\sinh 2 \pi d / L)^{2}}\right](\sin 4 \pi(x-C t) / L)+\left(\frac{\pi H}{L}\right)^{2} \cdot \frac{C}{2} \cdot \frac{\cosh 4 \pi(d+z) / L}{(\sinh 2 \pi d / L)^{2}} \cdot t$

and,

$$
\eta=\frac{H}{2} \frac{\sinh 2 \pi(d+z) / L}{\sinh 2 \pi d / L}(\cos 2 \pi(x-c t) / L)-\frac{3}{16} \cdot \frac{\pi H^{2}}{L} \frac{\sinh 4 \pi(d+z) / L}{(\sinh 2 \pi / L)^{4}}(\cos 4 \pi(x-c t) / L)
$$

Thus, the particle orbit lies a little above an ellipse at the crest and is a little flatter than an ellipse at the trough while, at the same time, the particle is moving forward (1.e., mass transport). This is shown in Fig. 18.

These equations show the most interesting result of the theory of stokes (1847). That is, by not neglecting the effect of height (the velocity of a particle depends not only upon its mean position, but also upon its displacement from 1ts mean position) it is shown that the particle velocity is greater in its forward 


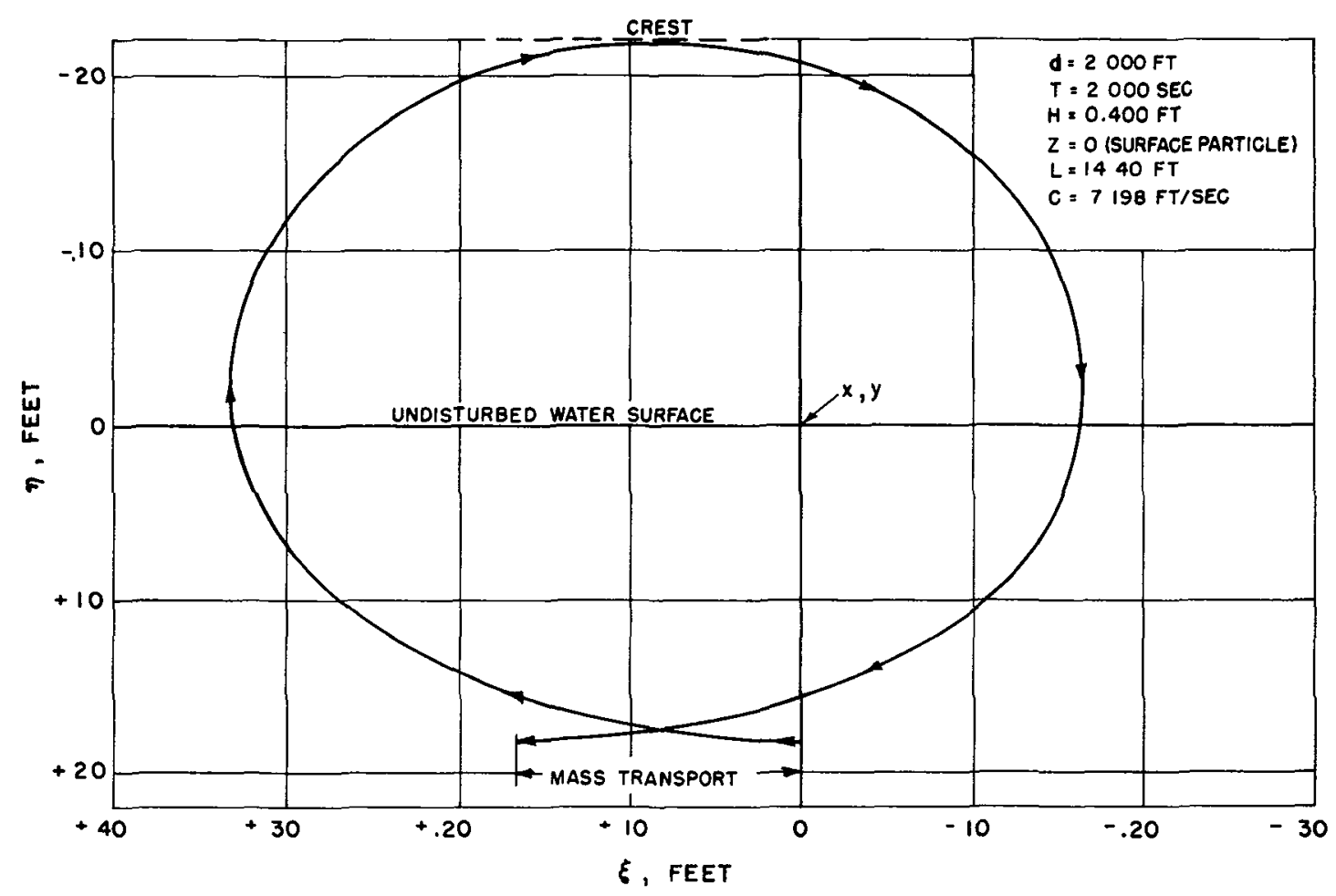

Fig. 18

Theoretical orbit of surface particle - Stokes' irrotational theory, second order.

movement (with the crest) than in its backward movement (with the trough). Laboratory experiments performed at the University of California, Berkeley, conf1rm this conclusion. This results in the fact that the forward motions of the particles are not altogether compensated by their backward motions. Hence, in addition to their orbital motion, there is a progressive motion in the direction of propagation of the waves. The orbits are open, not closed (Figs. 6 and I8). This motion has become known as "mass transport" and is given to the second approximation by

$$
\bar{U}=1 / 2(\pi \mathrm{H} / \mathrm{L})^{2} \mathrm{c} \frac{\cosh 4 \pi(\mathrm{d}+\mathrm{z}) / \mathrm{L}}{\sinh 22 \pi \mathrm{d} / \mathrm{L}}
$$

For deep-water, this becomes,

$$
\bar{U}_{0}=\left(\pi H_{0} / L_{0}\right)^{2} C_{0} e^{4 \pi z / L}
$$

which is identical with the equation expressing the horizontal velocity remaining (due to rotation) after wave motion has been destroyed in the rotational trochoidal theory (Equation 3I). In other words, in order for a wave of finite height to exist, it is necessary for this additional velocity to exist. In the trochoidal theory, it is in the form of molecular rotation (which is not substantiated by observations) of particles moving in a closed orbit, while, in the irrotational theory, it results from particles moving in an open orbit (which is substantiated by observations (Beach Erosion Board, 1941; Mitchim, 1940; Morison, 1948 ).

Maximum Theoretical Wave Steepness. Stokes (1847) came to the conclusion that for any wave whose crest angle was greater than 1200, the series would cease to be convergent and hence the wave form would become discontinuous. However, the possibility of a wave existing with a crest angle equal to 1200 was not shown until later. Michell (1893) found the theoretical limit was $\mathrm{H} / \mathrm{L}=0.14$ and Havelock (1918) found it to be 0.1418 . 
In nature, an infinitely long series of waves does not exist; rather a train consisting of a finite number of waves, which are formed by winds in a storm area, travels on the ocean surface. These "wave groups" travel at a different velocity than that of the individual waves. Rather simple examples of wave groups are waves generated at the bow of a ship and the waves generated in a wave tank by operating the wave generator for only a few strokes (Beach Erosion Board, 1942). In these cases, it can be seen that the lead wave in the group decreases in height as it progresses, the potential energy being transformed into kinetic energy as the wave form induces corresponding velocities in the previously undisturbed water. The wave finally disappears while, at the same time, a new wave begins to appear at the rear of the group as the velocity pattern left behind is such that the flow converges towards one section and diverges from another section, forming the crest and trough.

The velocity with which the wave group travels (Lamb, 1932) is given by,

$$
\mathrm{C}_{\mathrm{g}}=1 / 2 \mathrm{C}[1+(4 \pi \mathrm{d} / \mathrm{L}) / \sinh 4 \pi \mathrm{d} / \mathrm{L}]
$$

for waves of very small amplitude in any depth of water. The group velocity, as related to deep-water velocity, has been presented in Fig. 6, chapter 3. For deepwater, Equation 38 a becomes,

$$
\left(c_{g}\right)_{0}=1 / 2 c_{0}
$$

Reynolds (1877), for waves in infinite depth of water, and Rayleigh (1877), for waves in finite depth, developed equations for the transmission of energy by a wave group. In recent literature, the equations have been interpreted to mean that elther (a) all the energy advances with group velocity or (b) half the energy advances with the wave-front veloc1ty. However, as Rayleigh (1877) pointed out, for deep-water conditions :

\footnotetext{
"It appears that the energy propagated across any point, when a train of waves is passing, is only one-half of the energy necessary to supply the waves which pass in the same time, so that if the train of waves be limited, it is impossible that its front can be propagated with the full velocity of the waves .... because this would imply the acquisition of more energy than can in fact be supplied."
}

Reynolds (1877) states:

"So that after the waves have advanced through two wave-lengths the distribution of the energy will have advanced one, or the speed of the groups is one-half that of the waves."

From the mathematical arguments of these two investigators, it appears that the energy travels at the group velocity.

\section{REFERENCES}

Airy, G.B. (1845). On tides and waves: Encylopaedia Metropolitana, vol. 5 (mixed sciences), London, pp. 241-396.

Beach Erosion Board (1941). A study of progressive oscillatory waves in water: Tech. Report No. 1, Corps of Engineers, Washington, D.C.

Beach Erosion Board (1942). A sumnary of the theory of osclilatory waves: Tech. Report No. 2, Corps of Engineers, Washington, D.C.

Chinn, A.J. (1949). Effect of surface tension on wave velocity in shallow water: Tech. Report HE 116-302, Institute of Engineering Research, University of Califormia, Berkeley, California, (unpublished). 


\section{COASTAL ENGINEERING}

Folsom, R.G. (1947). Sub-surface pressures due to osc1llatory waves: Trans. Amer. Geophys. Union, vol. 28, pp. 875-881.

Folsom, R.G. (1949). Measurement of ocean waves: Trans. Amer. Geophys. Union, vol. $30, \mathrm{pp} .691-699$.

Froude, W. (1862). On the rolling of ships: Trans. Institute of Naval Architects, vol. $3, \mathrm{pp} .45-62$.

Gaillard, D.D. (1935). Wave action in relation to engineering structures: Reprinted at the Engineer School, Fort Belvoir, Virginia.

Gerstner, F. (1802). Theorie der wellen: Abhandlungen der Koniglichen Böhmischen Gesellschaft der Wissenschaften, Prague; also, Gilbert's - Annalen der Physik, vol. 32 , pp. $412-445$.

Havelock, E.T. (1918). Perlodic 1rrotational waves of finite helght: Proc. Royal Soc., London, Series A, vol. 95, pp. 38-51.

Hough, S.S. (1896). On the influence of viscosity on waves and currents: Proc., London Mathematical Society (I), vol. XXVIII, pp. 264-288.

Isaacs, J.D. and Wiegel, R.L. (1950). The thermopile wave meter: Trans. Amer. Geophys. Union, vol. 31, pp. 711-716.

Kaplan, K. (1950). Idealized model studies of the motion of surface waves: Tech. Report HE 116-316, Institute of Engineering Research, University of California; Berkeley, Califormia, (unpublished).

Lamb, H. (1932). Hydrodynamics, Sixth Edition, Cambridge Univ. Press.

La Place, P.S. (1775-76). Recherches sur quelques points du systéme du monde: Mem. Ac. Royal Soc., pp. 542-551.

Levi-Clvita, T. (1925). Determination rigoureuse des ondes d'ampleur finie: Math. Annalen, vol. 93, pp. 264-314.

Manning, G.C. (1939). The motion of ships among waves: Principles of Naval Architecture, vol. II, Edited by Tossell and Chapman.

Michel1, J.H. (1893). On the highest waves in water: Philosophical Magazine, (5), vol. XXXVI, pp. 430-437.

Mitchim, C.F. (1940). Oscillatory waves in deep water: The Military Engineer, vol. 32, pp. 107-109.

Morison, J.R. (1948). Wave pressures on structures: Master of Science thesis in Engineering, University of California, Berkeley, California, (unpublished).

Morison, J.R. (1951). The effect of wave steepness on wave velocity: Trans. Amer. Geophys. Union, vol. 32, pp. 201-206.

Rankine, W.J.M. (1863). On the exact form of waves near the surface of deep water: Philosophical Transactions of the Royal Society, London, pp. 127-138.

Rayle1gh, Lord (1877). On progressive waves: Proc., London Mathematical Society, vol. IX, pp. 21-26.

Reynolds, 0. (1877). On the rate of progression of groups of waves and the rate at which energy is transmitted by waves: Nature, vol. 16, pp. 343-344. 
Stokes, G.G. (1847). On the theory of oscillatory waves: Trans., Cambridge Philosophical Soclety, vol. VIII, p. 44I, and Supplement Scientific Papers, vol. I, p. 314 .

Struik, D.J. (1926). Determination rigoureuse des ondes irrotationeles periodiques dans un canal a profondeur finie: Math. Annalen, vol. 95, pp. 595-634.

Wlegel, R.L. (1948). Oscillatory waves: Bulletin of the Beach Erosion Board, Special Issue No. 1, Corps of Englneers, Washington, D.C.

Wiege1, R.L. (1950). Experimental study of surface waves in shoaling water: Trans. Amer. Geophys. Union, vol. 31, pp. 377-385. 